\title{
O AGENTE COMUNITÁRIO DE SAÚDE NA PROMOÇÃO DA SAÚDE DO HOMEM: POSSIBILIDADES E DESAFIOS
}

\author{
Claudia Moreira de Lima ${ }^{1}$ \\ Fernanda Almeida da Silva ${ }^{2}$ \\ Dayane dos Santos Souza Magalhaes ${ }^{3}$ \\ Rayanni Bruna Campos Ferro 4 \\ Grazielly Nogueira Xavier do Nascimento ${ }^{5}$ \\ Amanda Pereira de Siqueira ${ }^{6}$ \\ Paula Fernanda Albonette de Nóbrega ${ }^{7}$
}

RESUMO: O Agente comunitário de Saúde (ACS) desempenha papel fundamental no que se refere a atenção integral à saúde do homem, desenvolvendo ações voltadas a garantir o acesso deste público aos serviços de saúde, melhorando o acolhimento e visando as necessidades dos homens que serão atendidos pelo Sistema Único de Saúde (SUS) identificando necessidades em domicílio e peridomicílio. Objetivou-se com o estudo identificar se os Agentes comunitários de saúde (ACS's) realizam busca ativa em ações de saúde direcionadas ao homem na atenção primaria de saúde. Trata-se de uma pesquisa descritiva, exploratória, com abordagem qualitativa, realizada em novembro de 2019, com 31 ACS's pertencentes a estratégias de saúde da família localizadas num município da região médio norte de Mato Grosso. Para coleta de dados, utilizou-se questionário semiestruturado, do tipo autoaplicável. As análises foram apoiadas na análise de conteúdo de Bardin. Evidenciou-se que os ACS's possuem conhecimento acerca da importância da atenção direcionado a saúde do homem bem como da Política Nacional de Atenção Integral a Saúde do Homem - PNAISH, entretanto há barreiras que impossibilitam uma assistência integral a este público. Conclui-se que apesar das dificuldades encontradas, estes profissionais se

\footnotetext{
${ }^{\text {I }}$ Enfermeira Mestra, pelo Programa de Pós-Graduação em Ambiente e Saúde da Universidade de Cuiabá (UNIC). Professora Assistente da Universidade do Estado de Mato Grosso - UNEMAT, Diamantino, Mato Grosso-Brasil. E-mail: cml_claudiamoreira@hotmail.com.

${ }^{2}$ Enfermeira graduada pela Universidade do Estado de Mato Grosso - UNEMAT, Diamantino, Mato GrossoBrasil.

3 Enfermeira Mestra, pelo Programa de Pós-Graduação em Ambiente e Saúde da Universidade de Cuiabá (UNIC). Enfermeira assistencial na Empresa Brasileira de Serviços Hospitalares no Hospital Universitário Júlio Muller da Universidade Federal do Mato Grosso/UFMT/EBSERH.

${ }_{4}$ Enfermeira assistencial na Empresa Brasileira de Serviços Hospitalares no Hospital Universitário Júlio Muller da Universidade Federal do Mato Grosso/UFMT/EBSERH.

5 Enfermeira assistencial na Empresa Brasileira de Serviços Hospitalares no Hospital Universitário Júlio Muller da Universidade Federal do Mato Grosso/UFMT/EBSERH.

${ }^{6}$ Enfermeira graduada pela Universidade do Estado de Mato Grosso - UNEMAT, Diamantino, Mato GrossoBrasil.

7 Bióloga e Doutora em Ecologia, Conservação e Manejo da Vida Silvestre. Secretaria de Estado de Educação/SEDUC-MT, Diamantino, Mato Grosso-Brasil.
} 
mostram presentes e atuantes no desenvolver de estratégias que possibilitem atender o público masculino, com acolhimento e proporcionando dentro do possível um atendimento mais completo possível.

Palavras Chaves: Agente Comunitário de saúde. Saúde do Homem. Trabalhador.

ABSTRACT: The Community Health Agent (CHA) plays a fundamental role with regard to comprehensie attention to men's health, developing actions aimed at ensuring the access of this public to helath services, improving reception and targeting the needs of men who will be served by Unified Health System (SUS) identifying needs at home and around the home. The objective of the study was to identify whether Community health agentes (CHA's) perform na active search in health actions directed at men in primary healt care. This is a descriptive, exploratory research, with a qualitative approach, carried out in November 2019, with 3I CHA's belonging to Family healt strategies located in a municipality in the northern middle region of Mato Grosso. For data collection, a semistructured questionnaire, produced by the researchers, was used. The analyzes were supported by Bardin's contente analysis. It was evidenced that the ACS's have knowledge about the importance of attention directed to men's health as well as the National Policy for Comprehensive Care for Men's Healht - PNAISH, however there are barriers that prevent comprehensive assistance to this public. Is is concluded that despite the difficulties encountered, these professionals are presente and active in the development of strategies that make it possible to serve the male audience, with welcoming and providing as complete a possible servisse as possible.

Keywords: Community health agent. Men's Health. Primary health care.

\section{INTRODUÇÃO}

O Agente comunitário de Saúde (ACS) atua sendo o responsável na promoção e prevenção dá saúde pela comunidade na sua área territorial, elaborando, mapeando e conduzindo à população à atenção primária, contribuindo na construção e no planejamento de ações voltadas à saúde. A assistência prestada pelo ACS deu início na década de 90 , sendo vistos então como facilitadores nas ações em foco, o primeiro nível de atenção à saúde (BRASIL,2013).

Estes profissionais são sujeitos participativos e proativos, conhecedores do território ao qual está inserido e dos problemas da comunidade, com potencialidade de crescimento e evolução tanto social quanto economicamente, com uma facilidade em observar as pessoas, as coisas, os ambientes, e sempre atuando de modo respeitoso e com ética perante a comunidade e aos profissionais (BRASIL, 2016).

Dentre suas funções, estão as visitas domiciliares que deve acontecer de forma regular, fazendo registros da população, visando um ato preventivo e resolutivo de problemas e patologias inseridas neste meio, com ações de proteção vacinal, pré-natal, 
dúvidas de exames e consultas, dentre outros, direcionando estas famílias à Estratégia de Saúde dá Família (ESF) ou a outros atendimentos se preciso (BRASIL, 2013).

É na visita domiciliar que o ACS realiza suas ações voltadas à cada membro dá família, e dentre elas estão: o grau de parentesco existentes entre os membros da família, os membros moradores na residência, à função de cada membro dá família exercida estando: nas divisões dás funções domésticas, no divisório de despesas, no indicatório daquele que é à base emocional e espiritual, como também os valores, os preconceitos, os costumes e à religiosidade, especialmente os que podem intervir no cuidado à saúde (TERÇAS et.al, 2015).

No que se refere à Saúde do Homem, tem que ser desenvolvidas ações de saúde voltadas a garantir o acesso do homem aos serviços de saúde, melhorando o acolhimento e visando as necessidades dos homens que serão atendidos pelo Sistema Único de Saúde (SUS) (BRASIL, 2016).

A atenção à Saúde do Homem foi aos poucos ganhando destaque, tendo sua primeira abordagem cientifica na década de 70 nos Estados Unidos. Nos anos 90 começaram discuções acercada saúde do homem de forma mais enfática, refletindo algumas características do ser saudável e do ser doente visando à saúde integral e de qualidade para esta população (GOMES, 20II).

Diante ao exposto, o Ministério da Saúde (MS) instituiu a Política Nacional de Atenção Integral à Saúde do Homem (PNAISH), com intuito de promover ações de saúde que contribuam expressivamente para a concepção da realidade individual masculina nos seus diversos contextos socioculturais e político-econômicos, visando o crescimento na expectativa de vida e a diminuição de morbimortalidade alarmante nesta classe (BRASIL, 2009).

Um estudo revelou que os homens que mais buscam os serviços de saúde têm características como: idade acima de 50 anos, casado, baixo grau de escolaridade, renda familiar média de um salário-mínimo, e tendo como o principal motivo de buscarem o serviço de saúde foi devido a doenças crônicas como hipertensão e/ou diabetes (PEREIRA, 2015).

Diante o exposto temos o ACS como um profissional de suma importância em ações de modo geral, especialmente à saúde do homem, onde este profissional está em maior contato com a família e com o próprio homem uma vez que está inserido dentro da comunidade durante sua jornada de trabalho diária, sendo, portanto, um facilitar no que se refere a à abertura do diálogo, trocas de experiências e ao acolhimento (BRASI L,2013).

Dentro desse contexto, torna-se necessário descrever a abordagem deste profissionais no que se refere à saúde do homem trabalhador, o que proporcionara estimulo a realizar buscas pelos usuários masculinos, fazendo com que estes usuários realizem atendimentos primários nas unidades de saúde tendo o auxílio e instrução do próprio ACS propiciando que este usuário queira ter o conhecimento dele próprio, curiosidades e ações em saúde que poderão estimular a procura pela prevenção e promoção a saúde do homem, assim como contribuindo para uma atuação em saúde completa por parte do profissional ACS. 


\section{MATERIAIS E MÉTODOS}

Trata-se de uma pesquisa qualitativa, descritiva e exploratória realizada na Atenção Primária de um município localizado no médio-norte do estado de Mato Grosso, Brasil. Participaram da pesquisa 3I ACS que foram identificados com nomes fictícios, com o intuito de manter o anonimato.

Foi realizada uma entrevista com o uso de um roteiro semi-estruturado produzido pelos pesquisadores. As informações foram gravadas, posteriormente transcritas e a nalisadas pela técnica de análise de conteúdo segundo Bardin. Dentre as várias modalidades de análise de conteúdo, foi utilizada a análise temática, esta tem como base o tema que está relacionado a uma afirmativa sobre algum assunto. A análise temática agrupa várias relações e pode ser apresentada graficamente por meio de uma palavra, frase ou resumo (MYNAIO, 20I0) (BARDIN, 20II).

Foram obedecidos neste estudo os preceitos éticos e legais de pesquisas que envolvem seres humanos de acordo com a resolução $466 / 12^{15}$, assim como a resolução $311 / 2007^{16}$ do Conselho Federal de Enfermagem, sobre pesquisa envolvendo seres humanos. Para os sujeitos deste estudo foi solicitado a assinatura do Termo de Consentimento Livre e Esclarecido (TCLE) em duas vias. Para garantir o anonimato, a ética, e o sigilo das informações coletadas, os nomes serão substituídos, estando as informações em poder exclusivo das pesquisadoras. O projeto foi aprovado pelo Comitê de Ética em Pesquisa da Universidade de Cuiabá (UNIC), sob o 13655419.9.0000.5165 e parecer número 3.480.677.

\section{RESULTADOS E DISCUSSÃO}

Os sujeitos participantes deste estudo são de uma totalidade de 3I participantes, sendo 26 participantes do sexo feminino, com idades entre 23 a 57 anos, e 5 participantes sendo do sexo masculino, estes com idades entre 24 a 45 anos.

Os resultados obtidos corroboram com os de outros estudos, a exemplo o de Oliveira (2012), onde a predominância de ACS's do sexo feminino caracteriza a inserção feminina na força de trabalho em saúde. Esse perfil pode estar relacionado com o papel de cuidador que a mulher desempenha na sociedade.

Com relação à idade, os autores ainda afirmam que os ACS's com mais idade tendem a deter um conhecimento maior acerca dos problemas da comunidade, com mais vínculo e laços de amizade, corroborando com este estudo, visto que a idade mínima dos sujeitos em estudo é de 24 anos e máxima de 45 anos.

Com relação ao tempo de estudo, identificou-se que 50\% possuem ensino médio completo e ensino superior completo com especialização em na área da Atenção Primária. Em relação ao tempo de atuação do ACS na ESF todos atuam a mais de I ano na função.

No que se refere ao nível de escolaridade, os participantes do estudo corresponderam às exigências da Lei no 10.507 de julho 2002, na qual estabelece que estes devem ter concluído pelo menos o Ensino Fundamental e haver concluído com aproveitamento o curso de qualificação básica para sua formação (BRASIL, 2002). 
No que diz a respeito da renda mensal, um total de I4 ACS's recebem menos de um salário-mínimo e 17 até 3 salários-mínimos. Em estudo realizado por Santos (20II), apresentou que, apesar da grande importância que o ACS assume dentro da equipe, incluindo o aumento significativo de trabalho e responsabilidade, ainda é discrepante a diferença salarial entre os membros da equipe. Sendo este profissional o elo entre os usuários com o Sistema Único de Saúde (SUS), que é a porta de entrada das redes de atenção à saúde (BRASIL, 2019).

Quanto à variável capacitação, no que se refere à saúde do homem, tema este abordado no referido estudo, a maioria dos participantes relataram que não receberam capacitação, ou seja, treinamento em relação a saúde do homem, dado mais preocupante ainda foi que alguns participantes relataram o desconhecimento do conteúdo abordado na PNAISH. Essa falha na comunicação pode ocorrer devido ao processo de qualificação deste profissional que ainda é desestruturado, fragmentado e, na maioria das vezes, insuficiente em conteúdo que colabore com competências necessárias para o adequado desempenho de seu papel (BORNSTEIN; STOTZ, 2008).

A saúde do homem em si envolve principalmente a PNAISH, que distingue na diretriz da promoção de ações de saúde que possam contribuir de maneira significativa na compreensão da realidade masculina, mostradas em suas várias conjunções socioculturais e político-econômicos, acatando diversos níveis de desenvolvimento desta população, organização dos locais de saúde, e os tipos de gestão de Estados e Municípios (BRASIL, 2013).

Portanto, a PNAISH detém em alcançar objetivos, sendo necessário para melhor promover o acesso da população masculina jovem as unidades de saúde no ato de promover saúde diariamente e anualmente, e em favor disto ela aborda eixos temáticos para melhores acolhimento e prevenção ao homem, constituindo no acesso e acolhimento, saúde sexual e saúde reprodutiva, paternidade e cuidado, doenças prevalentes na população masculina, prevenção de violências e acidentes (BRASIL, 2013).

E como comprimento dessas atribuições, são incluídas de acordo com a Lei no II.350/2006, de 05 de outubro, os imediatos desempenhos do ACS, que dentre as ações inclui o estímulo à participação da comunidade nas políticas públicas voltadas para a área da saúde, a realização de visitas domiciliares periódicas, no ato de estarem alertas a situações de risco à família e participação em ações que fortaleçam o elo entre o setor da saúde e outras políticas que gerem qualidade de vida a população (ANDRADE, 2017).

\section{O AGENTE COMUNITÁRIO DE SAÚDE E O ATENDIMENTO A POPULAÇÃO MASCULINA}

O papel do ACS é atuar na educação para a saúde, por meio de ações educativas visando a promoção da saúde e a prevenção de doenças/agravos, com esclarecimento de dúvidas e incentivando os cuidados da população.

Segundo Ávila (2007), o programa de ACS inicialmente criado no estado do Ceará em 1987, como parte do programa de emergência contra a seca, tinha como foco principal a redução da mortalidade materna e infantil e, mesmo depois de décadas a concepção de 
trabalhar a saúde focando a mulher ainda é bastante disseminada. Com a criação da PNAISH, a população masculina passou a fazer parte de suas atividades, que até então não tida como prioridade.

Diante dessa nova realizada, a assistência do ACS ao novo público da ESF implica numa abordagem diferenciada com vistas a qualidade da assistência, com orientações de promoção da saúde e a prevenção de patologias voltadas a este novo público. Esta relação entre o ACS e o público masculino dará resultados no método da confiança e o respeito para com ambos, tendo assim o cuidado a este público ainda fica muito restrito as visitas domiciliares, sendo neste momento muitas vezes o único de atendimento ao homem, devendo neste momento ser abordado todo o assunto necessário para o momento.

\footnotetext{
"De quanto em quanto tempo a pessoa procura a unidade de saúde, se realizou algum exame no ano. Orientamos quanto a importância de esta realizando alguns exames com o objetivo de prevenção de futuras doenças." (ACS $\left.{ }_{I}\right)$

"Geralmente quando o morador e único, ele e muito limitado as informações, mas processo sempre orientar sobre a saúde dele sim, se e fumante, ou usa álcool, ou outras drogas ilícitas, é mais complicado pois o mesmo já tem uma opinião formada e não aceita mudar." (ACS 2$)$.

"São feita todas as orientações sobre todos os exames em relação da sua saúde. Todas as informações em relação como esta e oriento sempre que é necessário ele se prevenir sobre todas as doenças.” ( $\left.\mathrm{ACS}_{3}\right)$.
}

Nos relatos acima podemos identificar a intenção dos ACS, em realizar uma abordagem divergente na assistência para com a população masculina. As falas apresentam enfoque para com o homem de maneira integralizada e humanizada, de modo que as entrevistas são realizadas de forma valorizada, inspecionando as queixas deste homem e as orientações dadas a esta população.

Os métodos do cuidado oferecidos pelos ACS resultam na importância das necessidades do cuidado a família, podendo estabelecer práticas dominantes, impondo as necessidades e os limites de vida da população, incluindo o homem em si, colaborando para o fortalecimento das ações da ESF juntamente a esta população assistida (ASSIS; SILVA, 2018). Os relatos a seguir expressa esta visão:

"Se suas vacinas estão em dia, se fez consulta recente, se tem o cuidado com a H.A e a D.I.A no seu dia a dia, se toma cuidados gerais de higiene em sua vida."(ACS 4).

"Eu como agente comunitário de saúde abordo o paciente me apresentada formalmente e pergunto se já fez os exames de rotina, se não eu oriento a fazer." (ACS 5).

"Qual idade, se é único morador, se tem ido à unidade nos últimos 6 meses. Oriento a procurar a unidade para realização de exames de rotina “ '(ACS 7). 
"Tento agir de forma mais natural possível, coleto informações como documentação pessoal, cadastro na unidade, situação de saúde. Oriento sobre os funcionamentos, horários da unidade e programas da unidade." (ACS 8).

Nesta abordagem os ACS trazem através de suas falas seus métodos de entrevistas, orientações, prevenções e falas através da procura por este público, sendo estas realizadas nas visitas domiciliares rotineiramente.

A partir disto, é favorável a realização de educação em saúde em prol a este público masculino e a sensibilização deles nas medidas de prevenção e orientação de patologias e no mantimento a saúde, para que os mesmos se tornem seus oportunos cuidadores (DOMINGUES et al, 2018).

A população masculina deve ter incentivos no comparecimento a ESF, através de idéias centradas e criativas que de modo aproximem este homem e com isso realizar mais visitas domiciliares (DOMINGUES et al, 2018).

A partir do momento em que o profissional ACS faz a procura do usuário há uma aproximação relevante, criando-se um vínculo entre ambos. $O$ vínculo formado entre usuário e a equipe proporciona o aumento de novas afinidades, favorecendo o acesso do homem trabalhador nos serviços de saúde. Beneficiando a visita domiciliar, gerando artifícios que permitam a aproximação destes usuários a ESF (DOMINGUES et al, 2018). Os relatos a seguir expõem esses fatos:

"[...] ajo de forma espontânea e mais informal criando um vínculo amigável, geralmente busco saber os hábitos do homem (sendo boas ou más), se tem doenças crônicas bem como o vínculo familiar. Faço orientação de quando buscar os nossos serviços, controle e prevenção das doenças crônicas e a acessibilidade ao serviço de saúde pública. ' (ACS 9)

"Como está sua saúde, seu cartão de vacina, se ele é hipertenso ou diabético são feitas as orientações, sempre tem campanha de saúde do homem e várias outras orientações “' (ACS Io).

"Como está a sua saúde, está com o cartão de vacina em dias, se tem feito exames de rotina, exames próstata (se mais de 40 anos), se é hipertenso ou diabético, verifica se está coma hiper dia e receita em dias." (ACS $\left.{ }_{I I}\right)$.

Esses discursos encontram-se em consonância com os objetivos e princípios da PNAISH, a qual visa orientar as ações e serviços de saúde para a população masculina, com integralidade e equidade, primando pela humanização da atenção, de forma integrada às demais políticas existentes, priorizando a atenção primária como porta de entrada (BRASIL, 2009).

Desta maneira, os profissionais de saúde necessitam de atuação no cuidado a saúde masculina com embasamento nos princípios de equidade, humanização e universalidade, por meio da PNAISH que atente a modificações impactantes desta população (MOREIRA;

CARVALHO, 20I6). 
A política em si favorece a junção da população masculina aos serviços de saúde, por meio das práticas realizadas do PNAISH nas ESF. No entanto, para que o mesmo se torne eficaz é essencial investimentos e cautela em âmbito local priorizando e executando táticas em saúde de acordo com o perfil epidemiológico de suas demandas populacionais com vistas a maior participação masculina (MOREIRA; CARVALHO, 2016).

É importante ressaltar que a fala dos ACS entrevistados agrupam ações na sua prática de trabalho cotidiana ou seja em sua área de atuação, embora não tenham sidos preparados para este enfoque que é a saúde do homem.

O ACS advém de valores que são altamente valorizados pela população, eles possuem facilidade em transmitir e repassar cuidados em saúde, otimizando o ingresso ao cuidado dos moradores e com isso o aumento da confiança da população na resolução de seus problemas, e esta aproximação é o que atribui contato aos indivíduos masculinos (ZOLINGUER, 20II).

Entretanto a ação do ACS no que concerne à saúde do homem ainda encontra resistências e fragilidades apontadas pelo ACS no que tange a promoção em saúde:

“ [... ]Todo homem não gosta de se cuidar, pois o mesmo acha que não precisa por isso quando chega a procurar o serviço de saúde muitos já estão em estado crítico, por isso a educação em saúde tem um papel importante na visita domiciliar do seu ACS, através da confiança." (ACS 15$)$

E com isso torna-se explícito que os homens são posicionados no polo do não cuidador, enquanto às mulheres é atribuído o lugar do cuidado (BERBEL et al, 2018).

No Brasil dados epidemiológicos de morbimortalidade apresentam que o gênero masculino é mais vulnerável a situações de risco pelo fato de terem comportamentos mais agressivos e rebeldes na saúde e por incidirem em maiores números de óbitos que as mulheres (BERBEL et al, 2018). A baixa adesão do homem é justificada por:

"[...] Uma parte reclama do tempo, não faltam serviço pra prevenção, apenas urgência e emergência“" (ACS $\left.{ }_{17}\right)$.

"[...] Digo geralmente o homem é o provedor, isso o faz ausente o dia todo de casa, impossibilitando o mesmo ter acesso a UBS ' (ACS 19 ).

"[...] o que interfere sempre é o tempo, pois como trabalha fora alguns, não tem tempo" '(ACS $2 \mathrm{I})$.

"[...] muitos que trabalham não vão se cuidar pelo simples fato de não querer faltar o serviço com medo de perder o emprego e muitos patrões não gostar" (ACS 23).

Para Alves (20II), as dificuldades nos atendimentos devem-se ao fato de que os homens têm como justificativa para a pouca adesão o fato de serem irresponsáveis e de serem o provedor da casa e cultivarem uma conduta machista, estando assim, em consonância com os relatos do nosso estudo. 
Há diversas dificuldades em trazer o público masculino a unidade de saúde, sendo necessário a realização de horários diferenciados a este público, sendo o horário noturno o de melhor atendimento (MAIA; MALAGUTI, 2017).

"[...] quando fazemos atendimento diferenciado eles vêem em massa. Digo diferenciado depois do expediente de trabalho, depois das 17:30hs. Que fazemos no novembro azul." (ACS 25)

Em relação ao funcionamento da UBS em horários alternativos para facilitar a busca por atendimento, esta pode vir a ser uma alternativa viável, pois os estudos de Gomes (2007) comprovam que os horários de funcionamento dos serviços públicos não atendem as demandas dos homens e os horários são inapropriados para esse público em especial.

Hodiernamente as consultas de rotina por parte dos homens não é uma realidade melhorada e engrandecida, dados apresentados pelo Ministério da Saúde-MS apontam que 31\% dos homens não incluem em sua rotina cuidados preventivos a saúde nos serviços de saúde, e 55\% não realizam a procura por não haver necessidade alguma e como resultado, grande parte deste público vão a óbito em decorrências de enfermidade patológicas, que de certo modo poderiam ter sido evitadas (BRASIL, 2019).

Informações apontadas pelo IBGE indicam que a população masculina vive em média sete ou oito anos a menos do que a população feminina, e em contrapartida a expectativa de vida dos homens teve um alcance de $7 \mathrm{r}$ anos, e entre as mulheres a expectativa é de 78 anos (BRASIL, 2019). Em relação às facilidades e dificuldades apontadas pelos entrevistados, foram selecionados os seguintes trechos:

"No momento só dificuldade, facilidade não temos nenhuma, pois nas visitas domiciliares dificilmente encontramos o homem, e quando encontramos dificilmente eles atendem a nossa abordagem " (ACS 24$)$.

"Dificuldade: muitos acham que não precisam e não vão precisar de uma unidade básica. Facilidade: quando você já tem a confiança do seu cliente, ele aceita tudo o que você sugere de boa, porque já existe o vínculo de ACS e cliente e isso favorece muito através da confiança que o mesmo nos deposita. Isso faz tudo valer a pena!” (ACS 23).

Assim como retratados nas falas dos ACS ainda são existentes inúmeros casos em que os profissionais de saúde como também os agentes, muitas das vezes não se encontram preparados para proporcionar serviços que de modo atraiam e aproxime este público masculino às ESF, que é o primeiro contato primordial destes encontros, sejam eles diários, semanais, mensal ou anual. $E$ isso se relaciona também entre os homens a questões culturais que inclui a masculinidade e como também a insuficiente presença dos homens nestes ambientes (SIMOES et al, 2013).

Esses fatos relatam também a mudança diária de profissionais ACS nas ESF tornando dificultoso o contato, a aproximação e a confiança perante os homens, por conta disto, realizam seus serviços por períodos pequenos de tempo de uma estratégia para outra no mesmo local em que residem ou até mesmo de um município para outro envolvendo a 
troca ou transferência destes profissionais, tornando dificultoso o contato com o público masculino. As falas a seguir apontam essas falhas:

"Falta mais capacitação, formas de abordagem e a própria resistência do homem" '(ACS 28).

"Homens em alguns casos são mais queixosos por não se importar ou ignorar o funcionamento da saúde pública, também há um certo preconceito da parte do homem quanto ao funcionamento de alguns serviços...” (ACS 29).

Considerando os resultados encontrados, percebe-se que os ACS's possuem conhecimento acerca da importância da atenção direcionado a saúde do homem, e que apesar das dificuldades, estes profissionais se mostram presentes e atuantes no desenvolver de estratégias que possibilitem atender o público masculino, com acolhimento e proporcionando dentro do possível um atendimento mais completo possível.

\section{CONSIDERAÇÕES FINAIS}

Os ACS's realizam de modo diferenciado a procura e busca pela população masculina, o que os tornam ainda mais atuantes e reconhecidos pelos gestores, profissionais da saúde e comunidade, por tentarem realizar abordagens que advêm de melhora a saúde do homem, apesar do insuficiente conhecimento que estes ACS's têm e relatam a saúde do homem.

Visto disto os ACS necessitam de conhecimento e treinamentos a saúde do homem, como também os profissionais de saúde que atuam nas ESF's que é o primeiro contato que este homem irá adquirir em suas rotinas como também o conhecimento primordial da PNAISH que é a política indagada ao homem, e que ainda a fraco conhecimento dos profissionais no conhecimento desta política.

A política em si já é existente a algum tempo pelo MS e é desconhecida pelos profissionais da saúde referente ao que ela traz e aborda no contexto ao homem que é de grande importância para todo este assunto que é a população masculina, mostrada nacionalmente, individual, coletivo, grupal, família e/ou comunidade.

Conclui-se então que a saúde do homem que ressalta o homem trabalhador necessita de ser mais reconhecida pela saúde e pelos profissionais que fazem parte deste contexto, de modo que assim estes homens de modo geral irão proporcionar o bem a si mesmos, e menores preocupações aos serviços de saúde, e serão inspirados a serem agentes de mudança.

\section{REFERENCIAS}

ALVES, Railda Fernandes; SILVA, Renata Pimentel; ERNESTO, Monalisa Vasconcelos; LIMA, Ana Gabriella Barros; SOUZA, Fabiana Maria. Gênero e saúde: o cuidar do homem em debate. Psicologia: teoria e prática, $13(3)$, 152-166. 2011. 
Á VILA, Maria Marlene Marques. O programa de agentes comunitários de saúde: o caso de Uruburetama. Rev. Ciência e saúde coletiva, v.30, 2007.

BARDIN, L. Análise de Conteúdo. I Edição. São Paulo: Edições 70; 2011.

BORNSTEIN, Vera Joana; STOTZ, Eduardo Navarro. Concepções que integram a formação e o processo de trabalho dos agentes comunitários de saúde: uma revisão da literatura. Ciência \& Saúde Coletiva, I3(I): 259-268, 2008.

BRASIL. Ministério da Saúde. Guia de Saúde do Homem para Agente Comunitário de Saúde (ACS). Rio de Janeiro: Ministério da Saúde, 2016. [Citado em: 20 nov 2019]. Disponível em: <https://central3.to.gov.br/arquivo/369121/.

BRASIL. Lei no 10.507 de 10 de julho de 2002. Cria a profissão e dá outras providências. Diário Oficial da União, io jul. 2002. [Citado em: 19 nov 2019]. Disponível em: <http://www.cofen.gov.br/lei-n-10507-de-Io-de-julho-de-2002_4I65.html.

BRASIL. Ministério da Saúde. Secretaria de Atenção à Saúde. Departamento de Ações Programáticas e Estratégicas. Política Nacional de Atenção Integral à Saúde do Homem: princípios e diretrizes. Brasília: Ministério da Saúde, 2008. [Citado em: o7 Agos 2019]. Disponível em:

http://bvsms.saude.gov.br/bvs/publicacoes/politica_nacional_atencao_homem.pdf

BRASIL. Ministério da Saúde. Política nacional de atenção integral a saúde do homem [recurso eletrônico] / Elza Berger Salema Coelho... [et al] - Florianópolis: Universidade Federal de Santa Catarina, 2018. [Citado em: io agos 2019]. Disponível em: http://portalarquivos2.saude.gov.br/images/pdf/2018/novembro/o7/livroPol--ticas2018.pdf

BERBEL, NAN. A problematização e a aprendizagem baseada em problemas: diferentes termos ou diferentes caminhos. Interface Comun Saúde Educ. 2:139-54, 1998.

COFEN - Resolução COFEN nº 3II/2007: Código de ética dos Profissionais de Enfermagem. Disponível em: <http://www.cofen.gov.br/resoluo-cofen-3112007_4345.html.

GOMES, Romeu. Saúde do homem em debate. Rio de Janeiro: Fiocruz, 20II.

GOMES, Romeu; NASCIMENTO, Elaine Ferreira; ARAÚJO, Fábio Carvalho. Por que os homens buscam menos os serviços de saúde do que as mulheres? As explicações de homens com baixa escolaridade e homens com ensino superior. Cadernos de Saúde Pública. V. 23, n. 3, p. 565-574, 2007.

IBGE. Instituto Brasileiro de Geografia e Estatística (IBGE). Pesquisa Nacional por Amostra de Domicílios. Um Panorama da Saúde no Brasil: acesso e utilização dos serviços, 
condições de saúde e fatores de risco e proteção à saúde. Rio de Janeiro: IBGE; 2008.

MAIA, Simone Maria de A; MALAGUTTI, Willian. As dificuldades de percepção do enfermeiro da atenção primária à saúde do homem. Revista HUPE, Rio de Janeiro. 15 (4):336342. 2016.

MENDONÇA, Vitor Silva; ANDRADE, Angela Nobre de. A Política Nacional de Saúde do Homem: necessidade ou ilusão? Psicologia Política. Vol.ıo (20); 215-226, 2010.

MOREIRA, Michelle Araújo; CARVALHO, Camila Nunes. Atenção Integral à Saúde do Homem: Estratégias utilizadas por Enfermeiras (os) nas Unidades de Saúde da Família do interior da Bahia. Sau. \& Transf. Soc., Florianópolis, v.7(3), p.121-132, 2016.

OLIVEIRA, Díla Timbó; FERREIRA, Paulo Jorge de Oliveira; MENDONÇA, Larissa Bento de Araújo; OLIVEIRA, Helenir da Silva. Percepções do agente comunitário de saúde sobre sua atuação na estratégia saúde da família. Cogitare Enferm. 17(I): 132-7, 2012.

PEREIRA, Márcia Maria Marques; CÉZAR, Edna Samara Ribeiro; PEREIRA, Vagna Cristina Leite da Silva; BRAGA, Luanna Silva; ESPÍNOLA, Lawrencita Limeira; AZEVEDO, Elisangela Braga. Saúde do Homem na Atenção Básica: análise acerca do perfil e agravos à saúde. Rev enferm UFPE on line., Recife, 9(supl. I):440-7, jan., 2015.

SANTOS, Karina Tonini dos; SALIBA, Nemre Adas; MOIMAZ, Suzely Adas Saliba; ARCIERI, Renato Moreira; CARVALHO, Maria de Lourdes. Agente comunitário de saúde: perfil adequado à realidade do Programa Saúde da Família? Ciência \& Saúde Coletiva, I6(Supl. I): 1023-1028 2011.

SANTOS, Kelly Oliveira; SANTOS, Edirlei Machado. Onde Estão os Homens? O que os distanciam ou os aproximam dos Serviços da Atenção Primária à Saúde. Semina cienc. biol. saúde; 38(I): 79-88, jan./jun 2017.

Silva KL, Rodrigues AT. Ações intersetoriais para promoção da saúde na Estratégia Saúde da Família: experiências, desafios e possibilidades. Rev Bras Enferm. 2010;63(5):762-9.

SIMÕES, AV; SAMPAIO, DMN; SANTOS, FA; DUARTE, ACS; BOERY, RNSO. Política Nacional de Saúde do Homem: implementação a partir do trabalho dos Agentes Comunitários de Saúde. Enferm. Foco. 4(2): II2-II4, 2013.

TERÇAS, A.C.P, et.al. Aperfeiçoamento em Saúde da Família: uma abordagem ao processo de trabalho do Agente Comunitário de Saúde em Tangará da Serra. Tangará da Serra: Editora Ideias, 2015. 